NBS

Eechnical Note

no. 224

\title{
COMPUTATION OF A MODIFIED FRESNEL INTEGRAL ARISING IN THE THEORY OF DIFFRACTION BY A VARIABLE SCREEN
}

L. C. Walters and I. R. Wait

U. S. DEPARTMENT OF COMMERCE NATIONAL BUREAU OF STANDARDS 


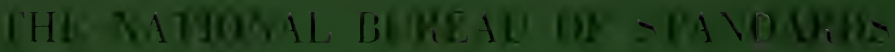

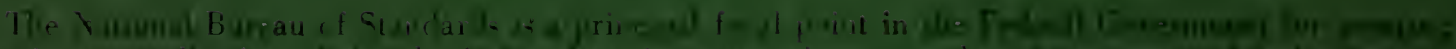

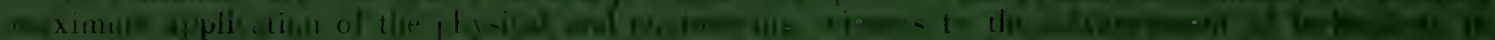

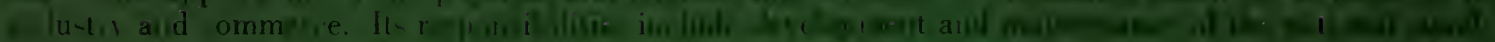

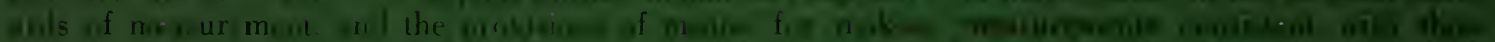

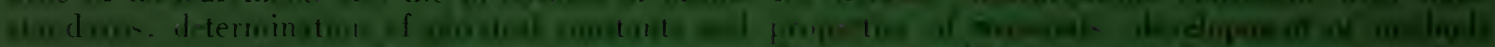

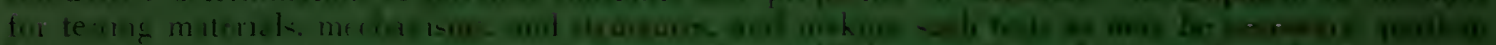

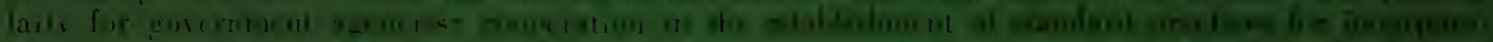

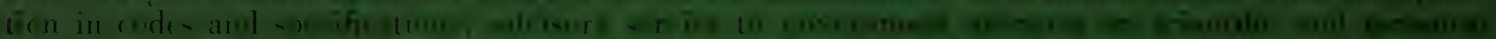

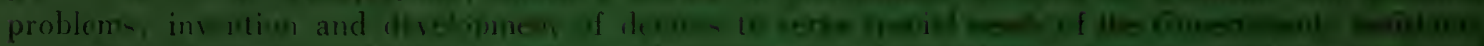

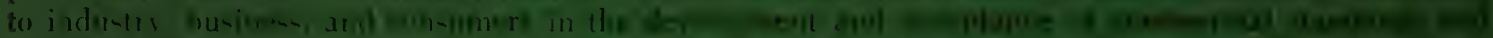

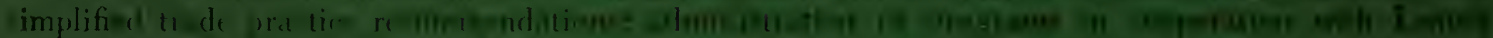

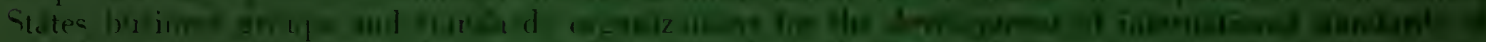

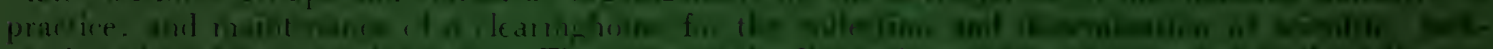

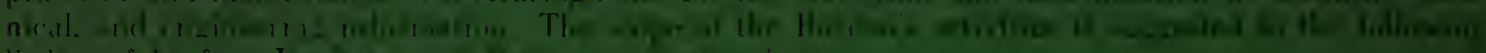

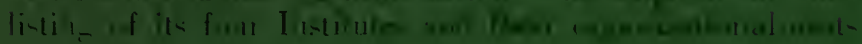

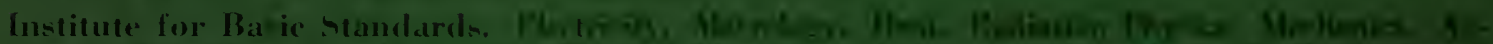

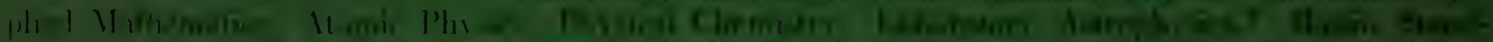

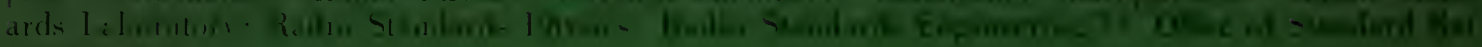
(e) I) 1

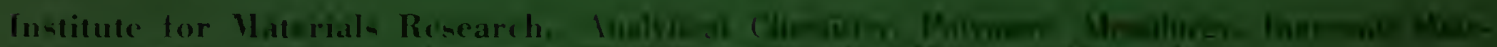
ii,l. Re

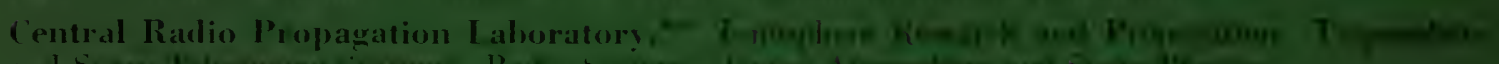

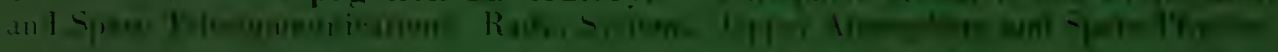

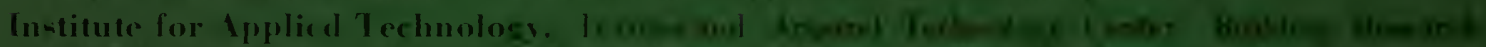

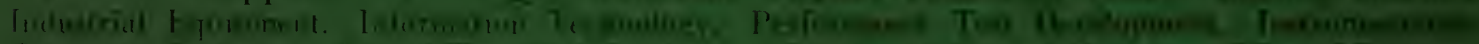

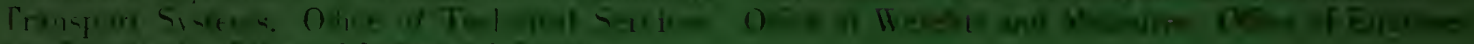

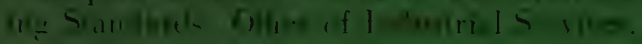

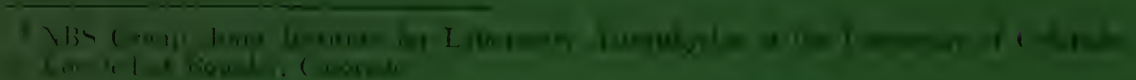




\title{
NATIONAL BUREAU OF STANDARDS
}

\section{Eechnical Mote 224 Issued October 14, 1964}

\section{COMPUTATION OF A MODIFIED FRESNEL INTEGRAL ARISING IN THE THEORY OF DIFFRACTION BY A VARIABLE SCREEN}

\author{
L. C. Walters and J. R. Wait \\ Central Radio Propagation Laboratory \\ National Bureau of Standards \\ Boulder, Colorado
}

\begin{abstract}
NBS Technical Notes are designed to supplement the Bureau's regular publications program. They provide a means for making available scientific data that are of transient or limited interest. Technical Notes may be listed or referred to in the open literature.
\end{abstract}

\footnotetext{
For sale by the Superintendent of Documents, U. S. Government Printing Office Washington, D.C. 20402

Price: 20 cents
} 

Abstract. ............................ 1

1. Introduction. ........................ 1

2. A Simple Physical Problem.................. 1

3. Various Aperture Functions and the Resultant Fields.................... 3

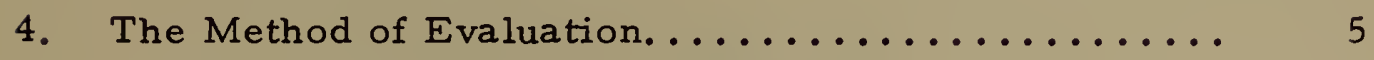

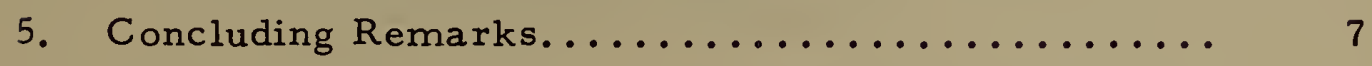

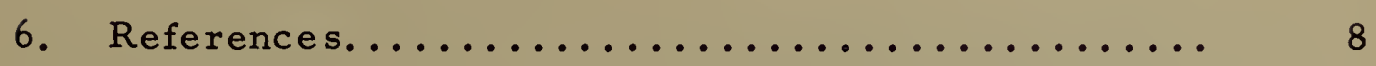

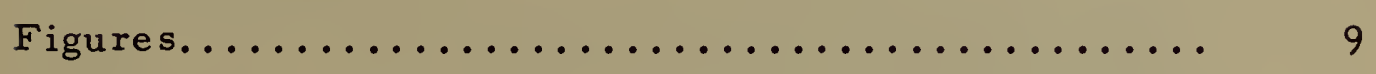

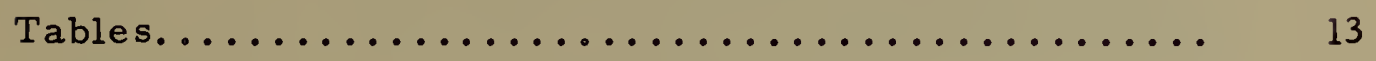

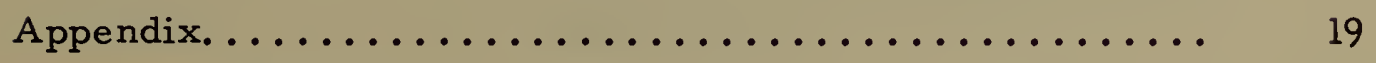

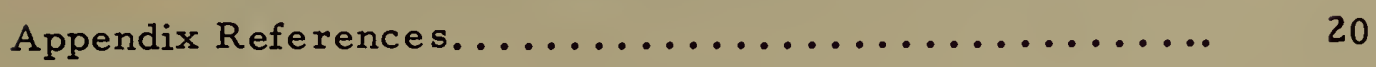





\section{C. Walters and J.R. Wait}

The integral of the form

$$
\int_{-\infty}^{+\infty} f(z) \exp \left(-i \pi z^{2} / 2\right) d z
$$

is considered in this note. The function $f(Z)$ varies from 0 to 1 in a monotonic fashion over the range of $\mathrm{Z}$. Some numerical results a re given which should be useful in certain diffraction and scattering problems involving objects of variable density.

\section{Introduction}

In numerous problems in the wave theory of diffraction, the solution is expressed approximately by expressions involving Fresnel integrals. These integrals, which have the form $\int \exp \left(-i \pi z^{2} / 2\right) d z$, are extensively tabulated for real limits. However, there is a class of problems involving non-opaque or partially transparent objects where functions of the form $\int f(Z) \exp \left(-i \pi Z^{2} / 2\right) d Z$ occur where $f(Z)$ is some non-slowly varying function. It is the purpose of this note to discuss this modified Fresnel integral for a class of functions $f(Z)$ which are monotonic in $Z$ over the range $-\infty$ to $+\infty$.

\section{A Simple Physical Problem}

To give the reader some insight, a simple physical model of diffraction by a variable screen is described to illustrate how such a modified Fresnel integral may arise. Thus, consider a thin planar slab (in the plane $\mathbf{x}=0$ of a Cartesian system $x, y, z$ ) of material whose permittivity differs from the surrounding medium. The 
permittivity (or the density in the equivalent acoustic problem) is assumed to be just a function of $y$, denoted $K(y)$. The slab is now illuminated by a plane wave from the negative $x$ direction. The observer, who is on the other side of the slab (i.e., positive $x$ ), will see a variation with $x$ of the field intensity $E(x)$ which bears some relation to the permittivity function $\mathrm{K}(\mathrm{y})$. Letting the observer be located at $y=0$, the application of first-order Born scattering theory leads readily to the result that [Born and Wolf, 1959]

$$
E(x) \cong \operatorname{const} x \int \frac{K(y)}{\rho^{\frac{1}{2}}} e^{-i k \rho} d y+E_{0},
$$

where $\rho=\left(x^{2}+y^{\dot{2}}\right)^{\frac{1}{2}}, k=2 \pi /$ wavelength, and $E_{0}$ is the field in the absence of the slab. Within this approximation, each line element of the screen is a re-radiating source for a cylindrical wave emanating from all points on the screen. The strength of these secondary source functions varies according to $K(y)$.

The scattered field, $E_{s}$, which is the difference $E(x)-E_{0}$, may be approximated in most cases by using the usual second-order phase approximation for the exponential factor exp $(-i k p)$. Thus,

$$
-i k \rho=-i k\left(x^{2}+y^{2}\right)^{\frac{1}{2}} \cong i k x-i \alpha y^{2}
$$

where $\alpha=k /(2 x)$. At the same time, $\rho^{\frac{1}{2}}$ in the denominator is replaced by $x^{\frac{i}{2}}$. It now follows that

$$
E^{s} \cong \text { const } \times \frac{e^{-i k x}}{x^{\frac{1}{2}}} \int_{-\infty}^{+\infty} K(y) \exp \left(-i \propto y^{2}\right) d y \text {. }
$$

On making a simple change of variable via $Z=(2 \alpha / \pi) y$, it is seen that 


$$
E \cong \text { const } \times \frac{e^{-i k x}}{x^{\frac{1}{2}}} F
$$

where

$$
F=\int_{-\infty}^{+\infty} f(Z) \exp \left(-i \pi Z^{2} / 2\right) d z
$$

and

$$
f(Z)=\text { const } \times K(y)
$$

In this normalization, the function $F$, which is the modified Fresnel integral, is proportional to the secondary field diffracted by the screen whose scattering properties vary according to the function $f(Z)$. The modified Fresnel integral, as given by (4), also arises in related diffraction problems such as occur in VLF propagation in the earth-ionosphere waveguide of variable width [Wait, 1964].

3. Various Aperture Functions and the Resultant Fields

The aperture function $f(Z)$ in the simplest case could be replaced by a step function. In other words, if

$$
f(Z)=u\left(Z-z_{o}\right)\left\{\begin{array}{lll}
=1 & \text { for } & Z>z_{0} \\
=0 & \text { for } & Z<z_{0}
\end{array}\right. \text {, }
$$

it is seen that

$$
F=\int_{Z_{0}}^{\infty} \exp \left(-i \pi Z^{2} / 2\right) d Z \text {, }
$$

which is the standard Fresnel integral. Physically, this would be applicable to the screen whose properties changed abruptly at $Z=Z_{0}$. Of more interest is the case when the aperture function $f(Z)$ changes gradually from 0 to $l$ about the central value $Z=Z_{0}$. 
Three aperture functions are considered. They are defined as follows, where $\beta$ is a constant:

I Linear Tapered Aperture

$$
\begin{array}{rlrl}
f(Z) & =\frac{1}{2}+\frac{\beta}{2}\left(Z-z_{0}\right) & \text { for } z_{0}-\frac{1}{\beta} \leq Z \leq Z_{0}+\frac{1}{\beta} \\
& =0 & & \text { for }-\infty \leq Z \leq z_{0}-\frac{1}{\beta} \\
& =1 & & \text { for } z_{0}+\frac{1}{\beta} \leq z \leq \infty
\end{array}
$$

II Cubic Tapered Aperture

$$
\begin{array}{rlrl}
f(Z) & =\frac{1}{2}+\frac{\beta}{2}\left(Z-Z_{0}\right)-\frac{2 \beta^{3}}{27}\left(Z-Z_{0}\right)^{3} & \text { for } Z_{0}-\frac{3}{2 \beta} \leq Z \leq Z_{0}+\frac{3}{2 \beta} \\
& =0 & & \text { for }-\infty \leq Z \leq Z_{0}-\frac{3}{2 \beta} \\
& =1 & & \text { for } Z_{0}+\frac{3}{2 \beta} \leq Z \leq \infty
\end{array}
$$

III Exponential Tapered Aperture

$$
f(Z)=\frac{1}{1+\exp \left[-2 \beta\left(Z-Z_{0}\right)\right]} \text { for }-\infty \leq Z \leq \infty
$$

The three forms of the function $f(Z)$ are shown in figure 1 where the ordinate is described as the "aperture blocking function." The term blocking, in this sense, refers to the partial filling of the aperture plane with scattering material.

The resulting behavior of the function $|F(z)|$, which is described as the "magnitude of the field", is illustrated in figures 2,3 , and 4 for the three respective forms of the aperture functions shown in figure 1. For large negative values of $z_{o}$ the aperture is totally 
effective, while for large positive values, the observer does not "see" the scattering material in the aperture. The case $Z_{0}=0$ could be described as a grazing condition. It is interesting to observe that certain intermediate values of $\beta$ result in a rapid decay of the field as a function of $\mathrm{Z}_{0}$.

\section{The Method of Evaluation}

The evaluation of the function $F(Z)$ for the aperture with a linear taper is quite straightforward. By a suitable change of variable and after integrating parts, it is readily found that, for case $I$, $F(z)=\left[\left(\frac{1}{2}-\frac{\beta Z_{0}}{2}\right) F_{0}(Z)+\frac{i \beta}{2 \pi} \exp \left(-i \frac{\pi}{2} Z^{2}\right)\right]_{Z_{1}}^{Z_{2}}+\frac{1-i}{2}-F_{0}\left(Z_{2}\right)$,

$Z_{2}=Z_{0}+(1 / \beta), \quad Z_{1}=Z_{0}-(1 / \beta)$ and $F_{0}(Z)=\int_{0}^{Z} \exp \left(-i \pi x^{2} / 2\right) d x$.

In a similar fashion, it is found for the cubic taper, namely, case II, that

$$
\begin{aligned}
F(z) & =\left\{\left(\frac{1}{2}-\frac{\beta Z_{0}}{2}\right) F_{0}(Z)+\frac{i \beta}{2 \pi} \exp \left(-i \pi Z^{2} / 2\right)\right. \\
& -\frac{2 \beta^{3} Z_{0}}{27}\left(\frac{i 3}{\pi}-Z_{0}^{2}\right) F_{0}(Z)-\frac{2 \beta^{3}}{27 \pi}\left(i Z^{2}+\frac{2}{\pi}-i 3 Z_{0} Z+i 3 Z_{0}^{2}\right) \\
& \left.\times \exp \left(-i \pi Z^{2} / 2\right)\right\}_{Z_{1}}^{Z_{2}}+\frac{1-i}{2}-F_{0}\left(Z_{2}\right)
\end{aligned}
$$

where

$$
z_{2}=z_{0}+\frac{3}{2 \beta}, z_{1}=z_{0}-\frac{3}{2 \beta}, \text { and } F_{0}(z)=\int_{0}^{Z} \exp \left(-i \pi x^{2} / 2\right) d x
$$


The case for the exponential taper cannot be expressed in simple form. Therefore, a numerical procedure was adopted. First, a lower limit $Z_{1}$ was chosen such that for $Z<Z_{1}$, there was a negligible contribution to the integral. Then, a corresponding upper limit was chosen such that for $Z>Z_{1}, f(Z)$ could be considered unity. For a specified accuracy, the ultimate choice of $Z_{1}$ and $Z_{2}$ was a function of $\beta$ and $Z_{0}$. For five-digit accuracy, it was found that $z_{2}=z_{0}+(7 / \beta)$ and $z_{1}=z_{0}-(7 / \beta)$. Thus,

$$
F(z) \cong \int_{Z_{1}}^{Z_{2}} f(Z) \exp \left(-i \pi Z^{2} / 2\right) d Z+\frac{1-i}{2}-F_{0}\left(Z_{2}\right) \text {, }
$$

where $f(Z)=\left\{1+\exp \left[-2 \beta\left(Z-Z_{0}\right)\right]\right\}^{-1}$.

The significance of the neglected portions of the integral could be verified by using the following asymptotic estimates:

$$
\int_{-\infty}^{Z} f(z) \exp \left(-i \pi z^{2} / 2\right) d z \sim \frac{f\left(z_{1}\right)}{\pi Z_{1}} \exp \left(-i \pi Z_{1}^{2} / 2\right),
$$

and

$$
\int_{Z_{2}}^{\infty} f(z) \exp \left(-i \pi z^{2} / 2\right) d z \sim \frac{f\left(z_{2}\right)}{\pi Z_{2}} \exp \left(-i \pi z_{2}^{2} / 2\right),
$$

which are strictly valid only when $z_{2} \rightarrow \infty$ and $z_{1} \rightarrow-\infty$.

The integral $\int_{Z_{1}}^{Z} f(Z) \exp \left(-i \pi Z^{2} / 2\right)$ d $Z$ was evaluated using a

Gaussian quadrature method. Following this standard technique (Kopal, 1955), the integral was approximated by a weighted sum of values of the ordinate. The required abscissas are roots of the 
Legendre polynomials. The number of intervals required for a given accuracy depend on $\beta$ and $Z_{0}$. For the values of $Z_{0}$ chosen here, $n=96$ was sufficient to obtain five-figure accuracy for $\beta=10,5$; while $n=192$ was required for $\beta=1,2,2.5,3$, and 4; and finally, $n=384$ was required for $\beta=0.5$. In the case $\beta=0.2$, it was necessary to modify the method because of poor convergence. Here, $n$ was fixed at 384 but $Z_{1}$ and $Z_{2}$ were selected so that the quadrature could be employed with the desired accuracy. For the regions $\mathrm{Z}<\mathrm{Z}_{1}$ and $\mathrm{Z}>\mathrm{Z}_{2}$, equations (12) and (13) were used to obtain the residual contributions. As a check, $Z_{1}$ and $Z_{2}$ were shifted by small amounts in order to detect any change in the final results.

The results of the calculations for all three aperture distributions are given in tables $1 \mathrm{a}, 1 \mathrm{~b}, 2 \mathrm{a}, 2 \mathrm{~b}, 3 \mathrm{a}$, and $3 \mathrm{~b}$ for $\mathrm{Z}_{\mathrm{o}}$ ranging from 0 to +5.0 . Results for negative values of $\mathrm{Z}_{0}$ can be obtained readily by the relation

$$
I\left(Z_{0}\right)+I\left(-Z_{0}\right)=1-i
$$

The accuracy of the se tables to the number of digits shown was checked by the method outlined in the appendix.

\section{Concluding Remarks}

It is believed that the results given here will be useful in various types of diffraction or scattering problems which involve objects with tapered distributions of density. A characteristic feature of the tapering is to remove some of the ripples associated with diffraction patterns from sharply bounded objects such as occur with the classical half-plane screen. Furthermore, the tapering of the density near the diffracting (or scattering) edge has the consequence of giving a 
relatively rapid transition in the diffraction from the illuminated region to the shadow region (with an analogous effect present in the scattering problem).

6. References

Born, M., and E. Wolf (1959), Principles of optics (Pergamon Press, Oxford).

Kopal, Z. (1955), Numerical analysis (John Wiley and Sons, Inc., New York, N. Y.).

Wait, J.R. (Feb. 1, 1964), On phase changes in very-low frequency propagation induced by an ionospheric depression of finite extent, J. Geophys. Res. vol. 68, No. 3, 441-445.

$-8-$ 


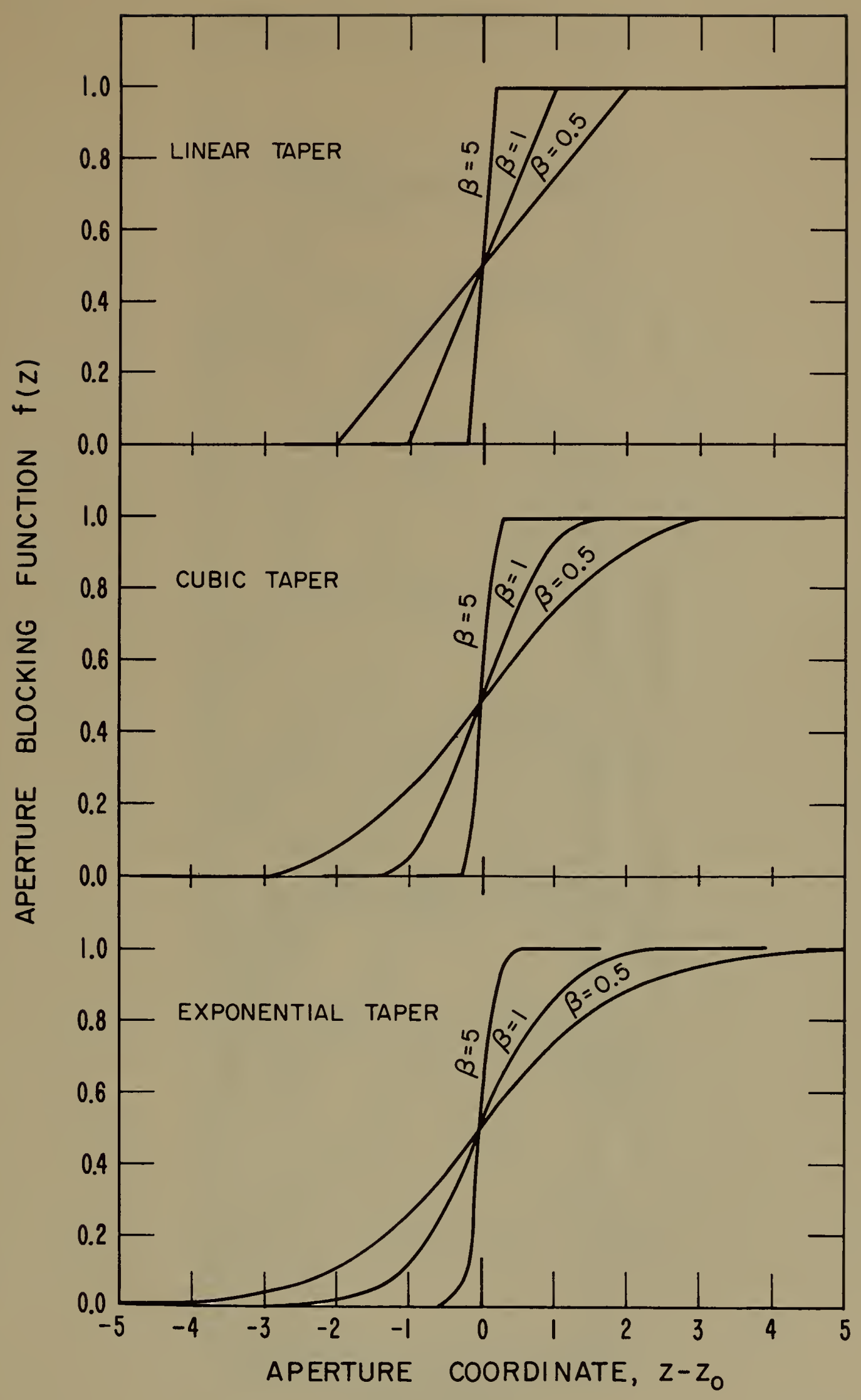

Fig. I 


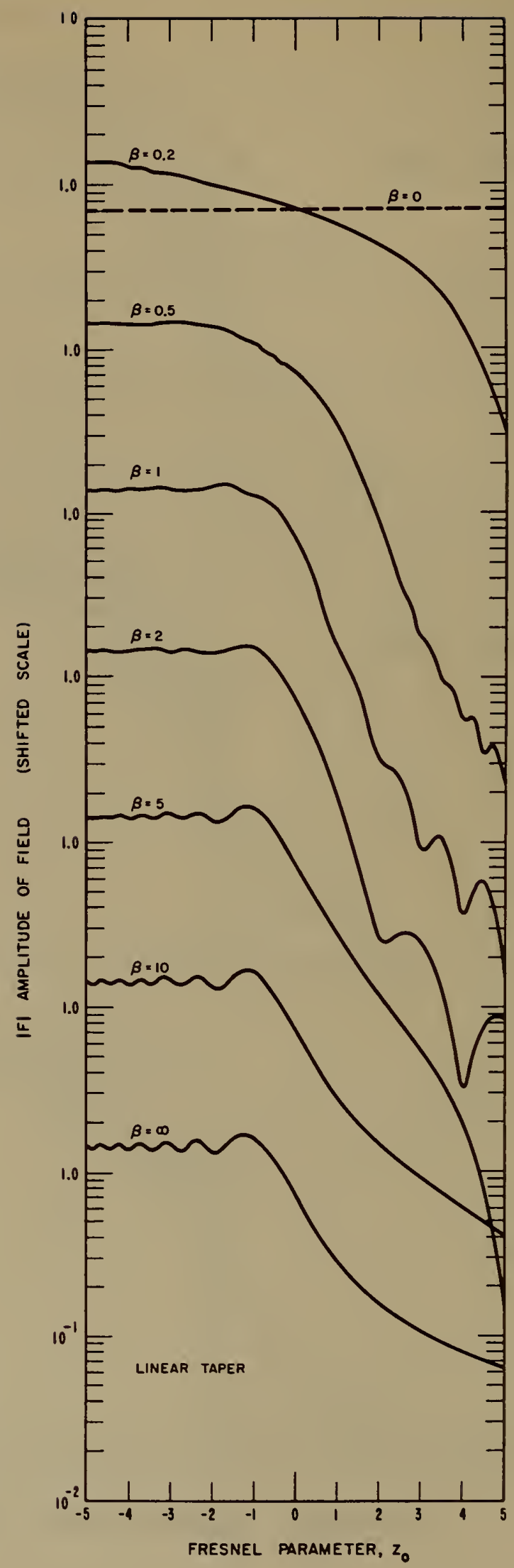

Fig. 2 


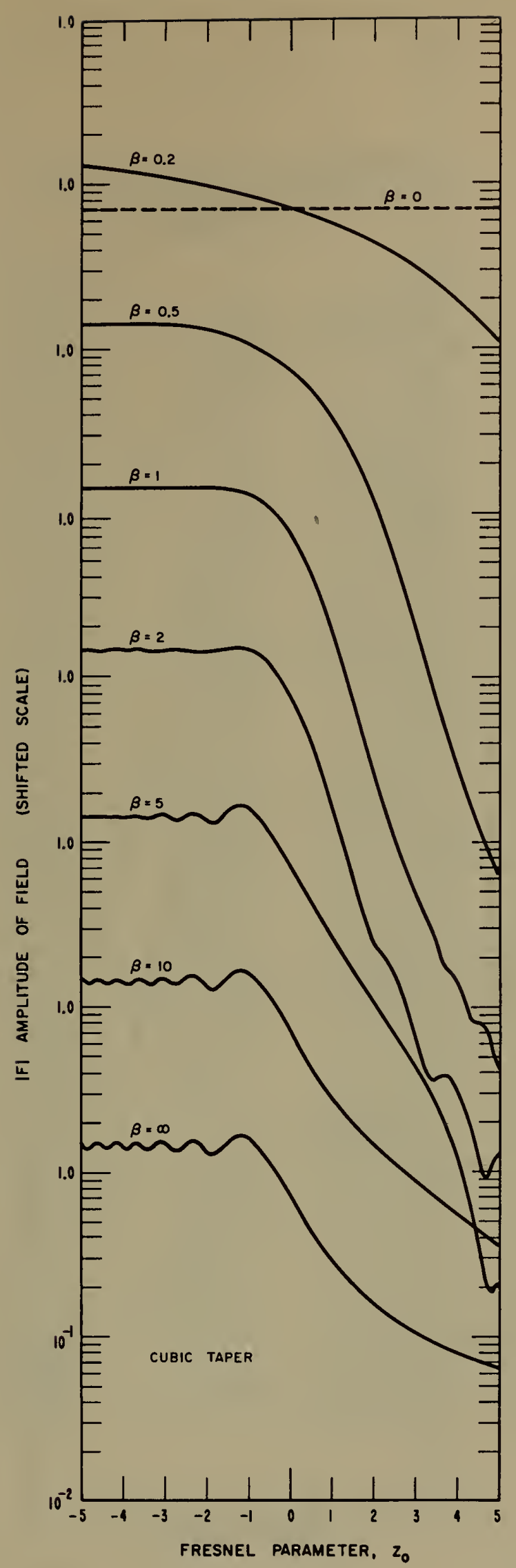

Fig. 3 


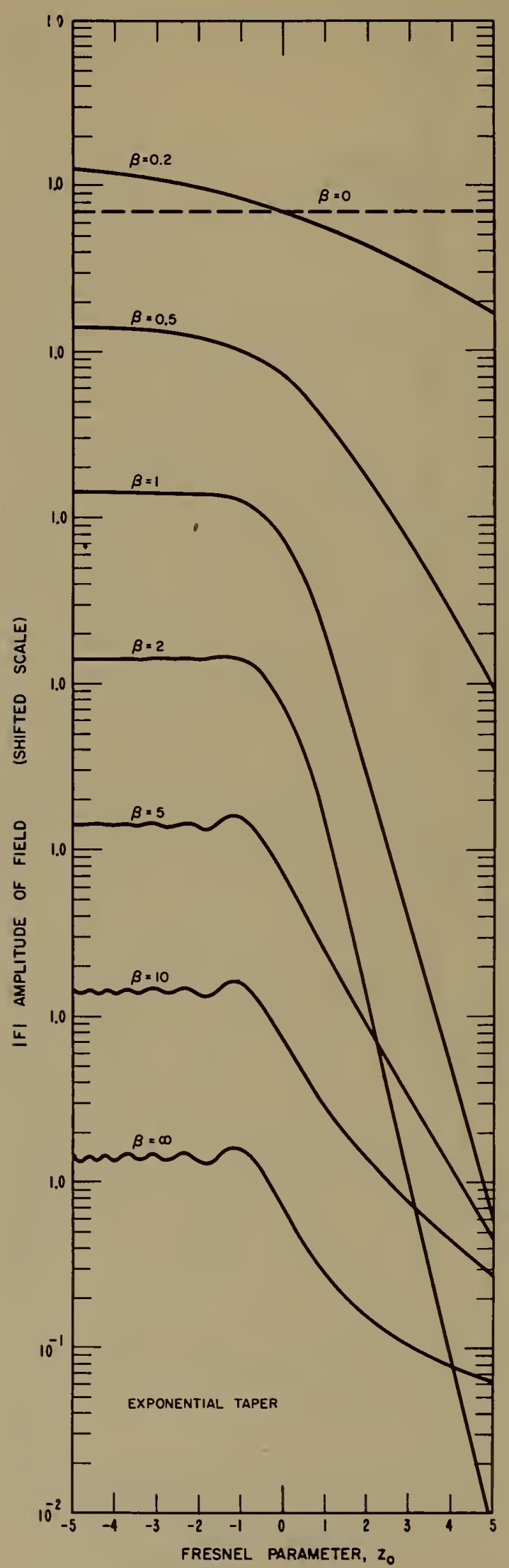

Fig. 4 
Table la

MODIFIED FRESNEL INTEGRAL LINEAR TAPERED APERTURE

F (REAL)

\begin{tabular}{|c|c|c|c|c|c|c|}
\hline$z_{0}$ & $B=0.20$ & $\beta=0.50$ & $\beta=1.00$ & $B=2.00$ & $\beta=5.00$ & $\beta=10.00$ \\
\hline 0. & 0.50000 & 0.50000 & 0.50000 & 0.50000 & 0.50000 & 0.50000 \\
\hline 0.1 & 0.48919 & 0.47559 & 0.42253 & 0.40173 & 0.40007 & 0.40001 \\
\hline 0.2 & 0.48000 & 0.45124 & 0.34813 & 0.30473 & $0.3 C 042$ & 0.30015 \\
\hline 0.3 & 0.47081 & 0.42721 & 0.27954 & 0.21048 & 0.20160 & 0.20083 \\
\hline 0.4 & 0.46002 & 0.40382 & 0.21888 & 0.12090 & 0.10475 & 0.10305 \\
\hline 0.5 & 0.44922 & 0.38109 & 0.16733 & 0.03842 & 0.01182 & 0.00867 \\
\hline 0.6 & 0.43991 & 0.35829 & 0.12492 & -0.03404 & -0.07421 & -0.07940 \\
\hline 0.7 & 0.43064 & 0.33390 & 0.09045 & -0.09327 & -0.14936 & -0.15710 \\
\hline 0.8 & 0.42022 & 0.30628 & 0.06175 & -0.13620 & -0.20871 & -0.21931 \\
\hline 0.9 & 0.40970 & 0.27482 & 0.03624 & -0.16052 & -0.24700 & -0.26033 \\
\hline 1.0 & 0.39965 & 0.24068 & 0.01175 & -0.16534 & -0.25936 & -0.27468 \\
\hline 1.1 & 0.38974 & 0.20655 & -0.01258 & -0.15190 & -0.24267 & -0.25840 \\
\hline 1.2 & 0.38036 & 0.17503 & -0.03576 & -0.12384 & -0.19691 & -0.21065 \\
\hline 1.3 & 0.37085 & 0.14697 & -0.05514 & -0.08698 & -0.12666 & -0.13542 \\
\hline 1.4 & 0.35992 & 0.12108 & -0.06745 & -0.04842 & -0.04184 & -0.04267 \\
\hline 1.5 & 0.34900 & 0.09554 & -0.07049 & -0.01492 & 0.04273 & 0.05173 \\
\hline 1.6 & 0.33955 & 0.07027 & -0.06455 & 0.00887 & 0.10963 & 0.12814 \\
\hline 1.7 & 0.33028 & 0.04718 & -0.05267 & 0.02174 & 0.14295 & 0.16752 \\
\hline 1.8 & 0.32074 & 0.02858 & -0.03931 & 0.02593 & 0.13373 & 0.15776 \\
\hline 1.9 & 0.31096 & 0.01419 & -0.02783 & 0.02562 & 0.08478 & 0.09995 \\
\hline 2.0 & 0.29989 & 0.00157 & -0.01863 & 0.02436 & 0.01244 & 0.01179 \\
\hline 2.1 & 0.28881 & -0.01073 & -0.00944 & 0.02281 & -0.05679 & -0.07500 \\
\hline 2.2 & 0.27900 & -0.02117 & 0.00193 & 0.01849 & -0.09556 & -0.12538 \\
\hline 2.3 & 0.26948 & -0.02729 & 0.01440 & 0.00836 & -0.08795 & -0.11605 \\
\hline 2.4 & 0.26065 & -0.02940 & 0.02329 & -0.00725 & -0.03919 & -0.05052 \\
\hline 2.5 & 0.25170 & -0.03024 & 0.02376 & -0.02235 & 0.02414 & 0.03768 \\
\hline 2.6 & 0.24131 & -0.03072 & 0.01553 & -0.02775 & 0.06648 & 0.09880 \\
\hline 2.7 & 0.23060 & -0.02858 & $0.0039 t$ & -0.01788 & 0.06393 & 0.09529 \\
\hline 2.8 & 0.21920 & -0.02260 & -0.00458 & 0.00288 & 0.02086 & 0.02906 \\
\hline 2.9 & 0.20789 & -0.01542 & -0.00786 & 0.02096 & -0.03082 & -0.05358 \\
\hline 3.0 & 0.19781 & -0.00957 & -0.00860 & 0.02315 & -0.05316 & -0.09070 \\
\hline 3.1 & 0.18813 & -0.00371 & -0.00887 & 0.00824 & -0.03147 & -0.05275 \\
\hline 3.2 & 0.17970 & 0.00334 & -0.00657 & -0.01067 & 0.01271 & 0.02775 \\
\hline 3.3 & 0.17143 & 0.00869 & 0.00056 & -0.01752 & 0.03930 & 0.07816 \\
\hline 3.4 & 0.16291 & 0.00990 & 0.00865 & -0.00847 & 0.02613 & 0.05085 \\
\hline 3.5 & 0.15410 & 0.00914 & 0.01002 & 0.00515 & -0.00973 & -0.02590 \\
\hline 3.6 & 0.14390 & 0.00791 & 0.00311 & 0.01000 & -0.02972 & -0.07030 \\
\hline 3.7 & 0.13323 & 0.00409 & -0.00448 & 0.00474 & -0.01514 & -0.03 .355 \\
\hline 3.8 & 0.12124 & -0.00150 & -0.00588 & -0.00175 & 0.01311 & 0.04003 \\
\hline 3.9 & 0.10894 & -0.00460 & -0.00301 & -0.00365 & 0.02085 & 0.05978 \\
\hline 4.0 & 0.09616 & -0.00517 & $-0.0005 c$ & -0.00311 & 0.00249 & 0.00166 \\
\hline 4.1 & 0.08337 & -0.00516 & 0.00201 & -0.00203 & -0.01467 & -0.05479 \\
\hline 4.2 & 0.07102 & -0.00264 & 0.00475 & 0.00170 & -0.00930 & -0.03100 \\
\hline $4 \cdot 3$ & 0.05894 & 0.00187 & 0.00297 & 0.00575 & 0.00652 & 0.03619 \\
\hline $4 \cdot 4$ & 0.04791 & 0.00374 & -0.00349 & 0.00246 & 0.00921 & 0.04404 \\
\hline 4.5 & 0.03733 & 0.00328 & -0.00547 & -0.00614 & -0.00110 & -0.01778 \\
\hline 4.6 & 0.02809 & 0.00224 & 0.00030 & -0.00597 & -0.00621 & -0.04625 \\
\hline 4.7 & 0.01939 & -0.00100 & 0.00428 & 0.00476 & -0.00121 & 0.00537 \\
\hline 4.8 & 0.01211 & -0.00348 & 0.00158 & 0.00769 & 0.00283 & 0.04383 \\
\hline 4.9 & 0.00540 & -0.00213 & -0.00134 & -0.00333 & 0.00167 & 0.00030 \\
\hline 5.0 & 0.00010 & -0.00028 & -0.00174 & -0.00782 & 0.00002 & -0.04050 \\
\hline
\end{tabular}


Table lb

MODIFIED FRESNEL INTEGRAL LINEAR TAPERED APERTURE

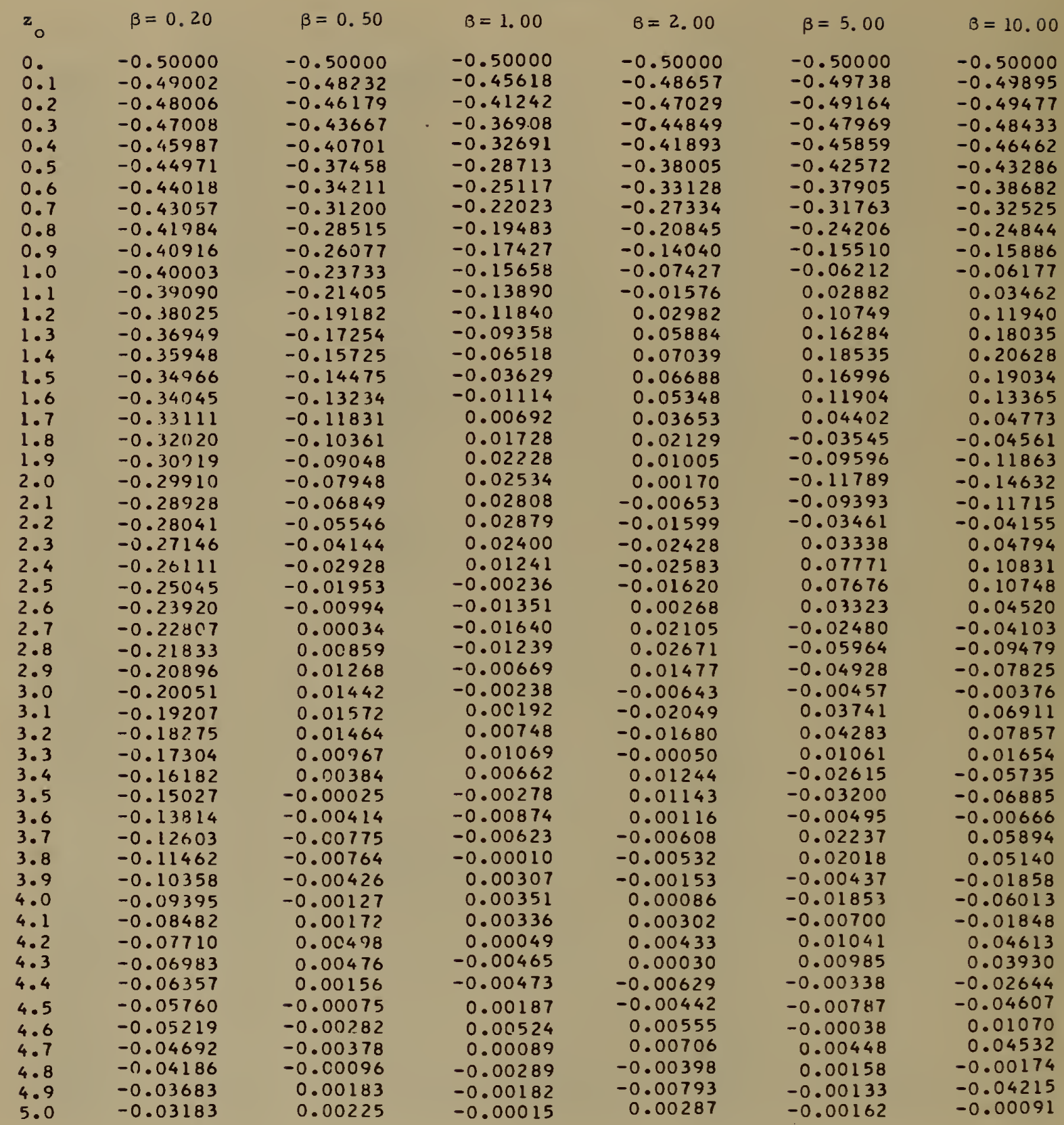


Table 2 a

MODIFIED FRESNEL INTEGRAL

CUBIC TAPERED APERTURE

F (REAL)

\begin{tabular}{|c|c|c|c|c|c|c|}
\hline$z_{0}$ & $\beta=0.20$ & $\beta=0.50$ & $B=1.00$ & $\beta=2.00$ & $\beta=5.00$ & $\beta=10.00$ \\
\hline 0.1 & $\begin{array}{l}0.50000 \\
0.48995\end{array}$ & $\begin{array}{l}0.50000 \\
0.47415\end{array}$ & $\begin{array}{l}0.90000 \\
0.43775\end{array}$ & $\begin{array}{l}0.50000 \\
0.40353\end{array}$ & $\begin{array}{l}0.50000 \\
0.40013\end{array}$ & $\begin{array}{l}0.50000 \\
0.40002 .\end{array}$ \\
\hline 0.2 & 3.11989 & 0.44837 & 0.17707 & 0.30865 & 0.30060 & 0.30018 \\
\hline 0.3 & 0.46985 & 0.42271 & 0.31926 & 0.21714 & 0.20204 & 0.20091 \\
\hline 0.4 & 0.45981 & 0.39720 & 0.25521 & 0.13105 & 0.10564 & 0.10324 \\
\hline 0.5 & 0.44979 & 0.37182 & 0.7 .1534 & 0.05278 & 0.01341 & 0.00903 \\
\hline 0.6 & 0.43979 & 0.34659 & $0.1636 \%$ & -0.01502 & -0.07169 & -0.07880 \\
\hline 0.7 & 0.42981 & 0.32164 & $0.12 \times 15$ & -0.06974 & -0.14568 & -0.15620 \\
\hline 0.8 & 0.41985 & 0.29722 & $0.0906 t$ & -0.10916 & -0.20379 & -0.21807 \\
\hline 0.9 & 0.40992 & 0.27354 & 0.05751 & -0.13195 & -0.24092 & -0.25877 \\
\hline 1.0 & 0.40003 & 0.25063 & 0.02914 & -0.13818 & -0.25252 & -0.27288 \\
\hline 1.1 & 0.39016 & 0.22825 & 0.00004 & -0.12963 & -0.23580 & -0.25653 \\
\hline 1.2 & 0.38035 & 0.30622 & -0.01150 & -0.10972 & -0.19108 & -0.20901 \\
\hline 1.3 & 0.37057 & 0.18453 & -0.02412 & -0.08312 & -0.12315 & -0.13436 \\
\hline 1.4 & 0.36083 & 0.16335 & -0.03235 & -0.05483 & -0.04183 & -0.04256 \\
\hline 1.5 & 0.35115 & 0.14279 & -0.03727 & -0.02911 & 0.03859 & 0.05069 \\
\hline 1.6 & 0.34152 & 0.12291 & -0.03051 & -0.00859 & 0.10166 & 0.12595 \\
\hline 1.7 & 0.33195 & 0.10389 & -0.1031124 & 0.00609 & 0.13278 & 0.16461 \\
\hline 1.8 & 0.322 .43 & 0.08603 & -0.23637 & 0.01570 & 0.12416 & 0.15486 \\
\hline 1.9 & 0.31299 & 0.06960 & -0.23111 & 0.02143 & 0.07908 & 0.09809 \\
\hline 2.0 & 0.30361 & $0.0546 E$ & $-0.0243 i$ & 0.02375 & 0.01311 & 0.01183 \\
\hline 2.1 & 0.27429 & $0 . r .4126$ & -0.21710 & 0.02214 & -0.04945 & -0.07288 \\
\hline 2.2 & 0.28506 & 0.02949 & $-0.01014 i$ & 0.01599 & -0.08426 & -0.12176 \\
\hline $2 \cdot 3$ & c. 27591 & $0 . ก 1943$ & $-0.00: 54$ & 0.00602 & -0.07783 & -0.11261 \\
\hline 2.4 & 0.26683 & 0.01697 & 0.00059 & -0.00485 & -0.03552 & -0.04908 \\
\hline 2.5 & 0.25784 & 0.00397 & 0.06471 & -0.01237 & 0.01901 & 0.03606 \\
\hline 2.6 & 0.74894 & -0.00156 & 0.00714 & -0.01344 & 0.05532 & 0.09488 \\
\hline 2.7 & 0.24014 & -0.00568 & 0.00745 & -0.00833 & 0.05373 & 0.09137 \\
\hline 2.8 & $0.7314 \%$ & $-0 . \operatorname{ccs} 864$ & 0.00605 & -0.00076 & 0.01861 & 0.02799 \\
\hline 2.9 & 0.22280 & -0.010 .53 & 0.20391 & 0.00477 & -0.02341 & -0.05084 \\
\hline 3.0 & 0.21430 & -0.01139 & 0.30165 & 0.00610 & -0.04187 & -0.08597 \\
\hline 3.1 & 0.20591 & -0.01141 & $-0.0005 \%$ & 0.00427 & -0.02560 & -0.04999 \\
\hline 3.2 & 0.19761 & -0.01081 & -0.100246 & 0.00153 & 0.00816 & 0.02595 \\
\hline 3.3 & $0.1894 ?$ & -0.00962 & -0.00316 & -0.00094 & 0.02881 & 0.07326 \\
\hline 3.4 & 0.18136 & -0.00801 & -0.00244 & -0.00291 & 0.02005 & 0.04762 \\
\hline 3.5 & 0.17343 & $-0.0062 z$ & -0.00096 & -0.00355 & -0.00547 & -0.02388 \\
\hline 3.6 & 0.16562 & -0.00432 & 0.00035 & -0.00166 & -0.02024 & -0.06509 \\
\hline 3.7 & 0.15793 & -0.00246 & 0.00125 & 0.00190 & -0.01131 & -0.03105 \\
\hline 3.8 & 0.15036 & -0.00085 & 0.30163 & 0.00371 & 0.00730 & 0.03652 \\
\hline 3.9 & 0.14293 & 0.00045 & 0.00113 & 0.00161 & 0.01327 & 0.05460 \\
\hline 4.0 & 0.13566 & 0.00139 & -0. nncois & -0.00196 & 0.00285 & 0.00169 \\
\hline $4 \cdot 1$ & 0.12854 & 0.00183 & $-0 . \operatorname{scos} 97$ & -0.00274 & -0.00778 & -0.04932 \\
\hline 4.2 & 0.12155 & 0.00188 & -0.00095 & -0.00035 & -0.00602 & -0.02791 \\
\hline 4.3 & 0.11469 & 0.00157 & -0.001143 & 0.00165 & 0.00217 & 0.03205 \\
\hline 4.4 & 0.10797 & 0.00098 & $0.0002 c$ & 0.00122 & 0.00475 & 0.03903 \\
\hline 4.5 & 0.10142 & 0.00034 & 0.00074 & -0.00014 & 0.00091 & -0.01555 \\
\hline 4.6 & 0.09506 & -0.00025 & 0.00062 & -0.00082 & -0.00211 & -0.04047 \\
\hline 4.7 & 0.08888 & -0.00066 & -0.00014 & -0.00080 & -0.00181 & 0.00451 \\
\hline 4.8 & 0.08286 & -0.100075 & -0.00058 & 0.00003 & -0.00021 & 0.03782 \\
\hline 4.9 & 0.07698 & -0.00061 & $-0.0003 / 4$ & 0.00113 & 0.00172 & 0.00039 \\
\hline 5.0 & 0.07125 & -0.0 .0026 & 0.00006 & 0.00057 & 0.00185 & -0.03460 \\
\hline
\end{tabular}


Table $2 \mathrm{~b}$

MODIFIED FRESNEL INTEGRAL CUBIC TAPERED APERTURE

F (IMAG)

\begin{tabular}{|c|c|c|c|c|c|c|}
\hline$z_{\circ}$ & $\beta=0.20$ & $\beta=0.50$ & $\beta=1.00$ & $\beta=2.00$ & $\beta=5.00$ & $\beta=10.00$ \\
\hline 0. & $\begin{array}{l}-0.50000 \\
-0.49006\end{array}$ & $\begin{array}{l}-0.50000 \\
-0.47621\end{array}$ & $\begin{array}{l}-0.500<0 \\
-0.45634\end{array}$ & $\begin{array}{l}-0.50000 \\
-0.48243\end{array}$ & $\begin{array}{l}-0.50000 \\
-0.49665\end{array}$ & $\begin{array}{l}-0.50000 \\
-0.49877\end{array}$ \\
\hline $\begin{array}{l}0.1 \\
0.2\end{array}$ & $\begin{array}{l}-0.49006 \\
-0.48012\end{array}$ & -0.45221 & -0.41378 & -0.46228 & -0.49019 & $\begin{array}{l}-0.49877 \\
-0.49440\end{array}$ \\
\hline 0.3 & -0.47019 & -0.42801 & -0.37331 & -0.43720 & -0.47755 & -0.48379 \\
\hline 0.4 & -0.46026 & -0.40387 & -0.33566 & -0.40533 & -0.45583 & -0.46391 \\
\hline 0.5 & -0.45036 & -0.38014 & -0.30121 & -0.36555 & -0.42748 & -0.43202 \\
\hline 0.6 & -0.44047 & -0.35699 & -0.25987 & -0.31770 & -0.37558 & -0.38591 \\
\hline 0.7 & -0.43060 & -0.33438 & -0.24112 & -0.26285 & -0.31429 & -0.32435 \\
\hline 0.8 & -0.42076 & -0.31214 & -0.21416 & -0.20331 & -0.23935 & -0.24768 \\
\hline 0.9 & -0.41094 & -0.29019 & $-0.18 \times 17$ & -0.14253 & -0.15364 & -0.15841 \\
\hline 1.0 & -0.40115 & -0.26858 & -3.16263 & -0.08471 & -0.06255 & -0.06179 \\
\hline 1.1 & -0.39141 & -0.24739 & -0.13757 & 0.03415 & 0.02598 & 0.03396 \\
\hline 1.2 & -0.38170 & -0.22602 & -0.11348 & 0.00562 & 0.10202 & 0.11801 \\
\hline 1.3 & -0.372 .04 & -0.20634 & -0.07109 & 0.03258 & 0.15509 & 0.17828 \\
\hline 1.4 & -0.36242 & -0.18676 & -0.07090 & 0.04675 & 0.17635 & 0.20378 \\
\hline 1.5 & -0.35285 & -0.16824 & -0.05295 & 0.05013 & 0.16147 & 0.18789 \\
\hline 1.6 & -0.34333 & -0.15096 & -0.03683 & 0.04600 & 0.11323 & 0.13187 \\
\hline 1.7 & -0.33387 & -0.13491 & -0.02244 & 0.03778 & 0.04288 & 0.04723 \\
\hline 1.8 & -0.32448 & -0.1200 .4 & -3.00975 & 0.02795 & -0.03097 & -0.04442 \\
\hline 1.9 & -0.31514 & -0.10640 & 0.0 .2053 & 0.01756 & -0.08671 & -0.11593 \\
\hline 2.0 & -0.30587 & -0.09401 & 0.00780 & 0.00678 & -0.10679 & -0.14289 \\
\hline 2.1 & -0.29667 & -0.08265 & $3.012 c 2$ & -0.00399 & -0.08529 & -0.11432 \\
\hline 2.2 & -0.28756 & -0.07214 & 0.01380 & -0.01312 & -0.03241 & -0.04064 \\
\hline 2.3 & -0.27851 & -0.06246 & 0.01385 & -0.01799 & 0.02766 & 0.04621 \\
\hline 2.4 & -0.26354 & -0.05359 & 0.01249 & -0.01652 & 0.06658 & 0.10458 \\
\hline 2.5 & -0.26067 & -0.04537 & 0.00367 & -0.00913 & 0.06618 & 0.10369 \\
\hline 2.6 & -0.25189 & -0.03770 & 0.00570 & 0.00065 & 0.02954 & 0.04368 \\
\hline 2.7 & -0.24319 & -0.03067 & 0.00157 & 0.00792 & -0.01905 & -0.03908 \\
\hline 2.8 & -0.23459 & -0.07429 & $-0 . \operatorname{col} 161$ & 0.00955 & -0.04824 & -0.09042 \\
\hline 2.9 & -0.22609 & -0.01846 & -0.00345 & 0.00616 & -0.04050 & -0.07461 \\
\hline 3.0 & -0.21771 & -0.01324 & $-0.00+21)$ & 0.00113 & -0.00521 & -0.00376 \\
\hline 3.1 & -0.20341 & -0.00874 & -3.00401 & -0.00244 & 0.02799 & 0.06515 \\
\hline 3.2 & -0.20122 & -0.00490 & -0.00273 & -0.00373 & 0.03298 & 0.07404 \\
\hline 3.3 & -0.19315 & -0.00172 & -0.00062 & -0.00348 & 0.00936 & 0.01570 \\
\hline 3.4 & -0.18522 & 0.00069 & 0.00125 & -0.00199 & -0.01792 & -0.05342 \\
\hline 3.5 & -0.17740 & 0.00233 & $0.00 \geq 05$ & 0.00074 & -0.02308 & -0.06398 \\
\hline 3.6 & -0.16969 & 0.00346 & 0.00186 & 0.00336 & -0.00485 & -0.00632 \\
\hline 3.7 & -0.16210 & 0.00386 & 0.00117 & 0.00328 & 0.01415 & 0.05420 \\
\hline 3.8 & -0.15465 & 0.00371 & 0.00007 & -0.00000 & 0.01381 & 0.04711 \\
\hline 3.9 & -0.14736 & 0.00318 & -0.00107 & -0.00312 & -0.00137 & -0.01675 \\
\hline 4.0 & -0.1402 .1 & 0.00231 & -2.07140 & -0.00254 & -0.01089 & -0.05458 \\
\hline 4.1 & -0.13318 & 0.00130 & -0.00067 & 0.00069 & -0.00521 & -0.01686 \\
\hline 4.2 & -0.12627 & $0.0 c 036$ & $(1.0 .01) 22$ & 0.00236 & 0.00472 & 0.04130 \\
\hline 4.3 & -0.11752 & -0.00046 & 0.00070 & 0.00098 & 0.00571 & 0.03513 \\
\hline 4.4 & -0.11295 & -0.00097 & 0.00076 & -0.00086 & -0.00029 & -0.02335 \\
\hline 4.5 & -0.10655 & -0.00113 & 0.00031 & -0.00112 & -0.00345 & -0.04077 \\
\hline 4.6 & -0.10031 & -0.00100 & -0.00048 & -0.00042 & -0.00157 & 0.00931 \\
\hline 4.7 & -0.09422 & $=0.00060$ & -0.00070 & 0.00043 & 0.00088 & 0.03957 \\
\hline 4.8 & -0.08826 & -0.00012 & -0.00015 & 0.00102 & 0.00182 & -0.00113 \\
\hline 4.9 & -0.08246 & 0.00029 & $0.00) 33$ & 0.00031 & 0.00114 & -0.03629 \\
\hline 5.0 & -0.07685 & 0.00054 & 0.90041 & -0.00116 & -0.00158 & -0.00088 \\
\hline
\end{tabular}




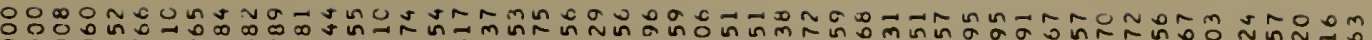

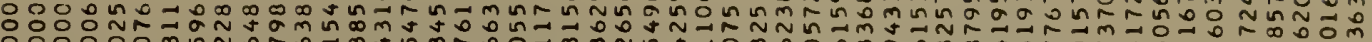

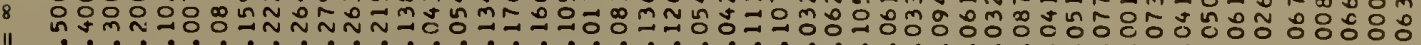

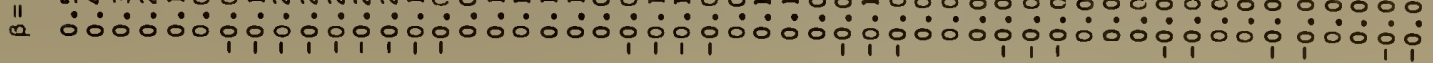

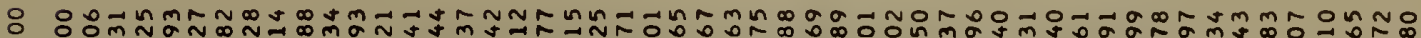

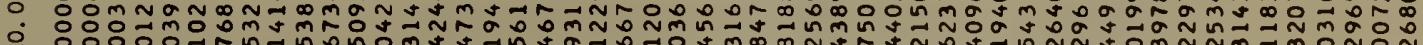

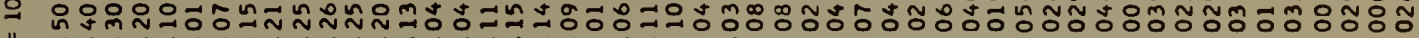

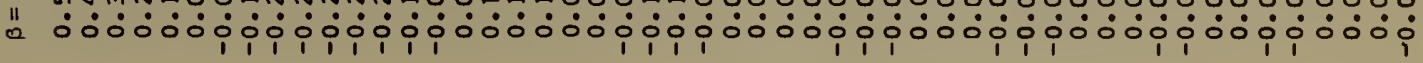

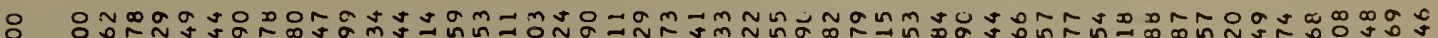

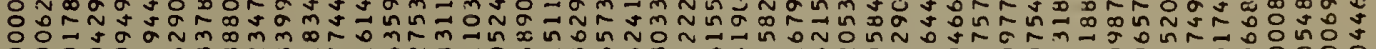

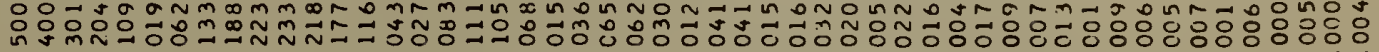

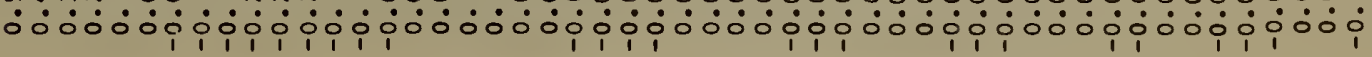

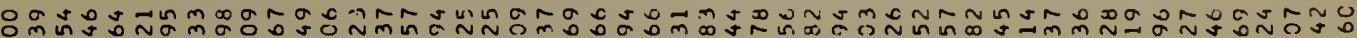

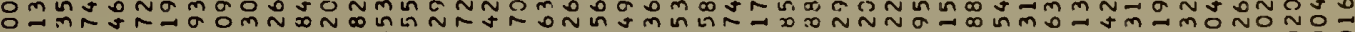

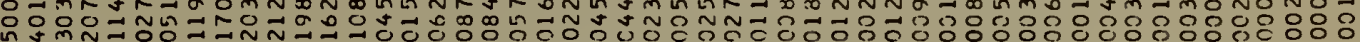
"l ó

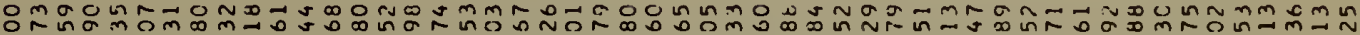

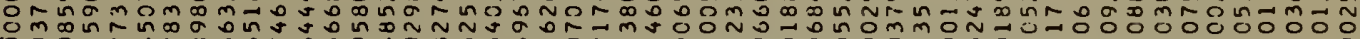

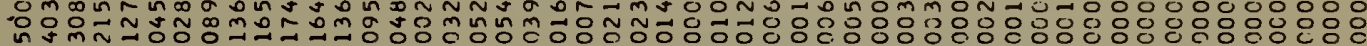

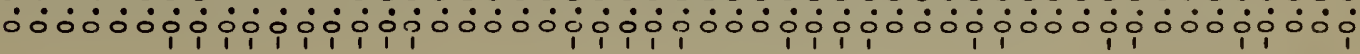

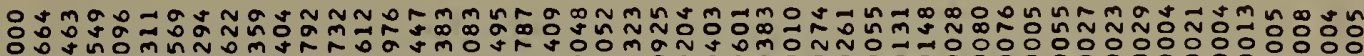

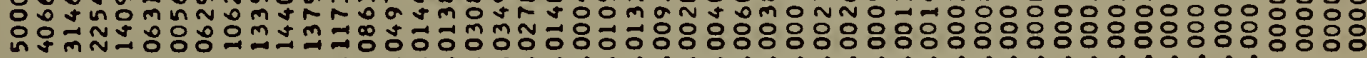

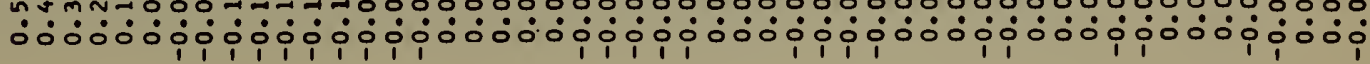

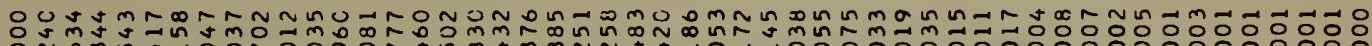

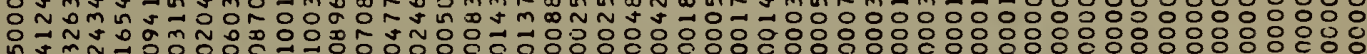

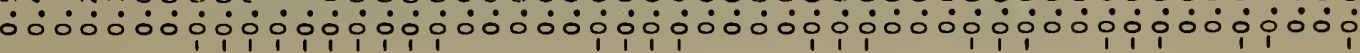

8

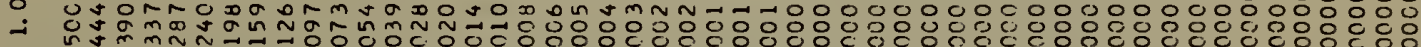
II ó ió

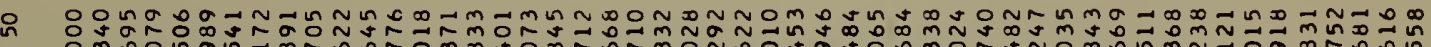

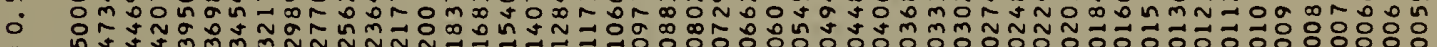
n

n 


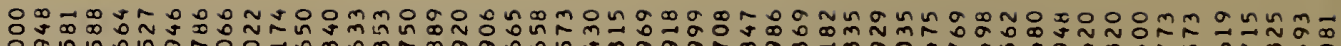

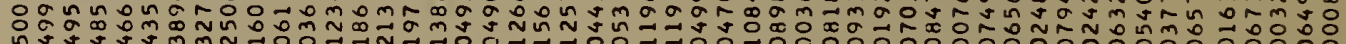

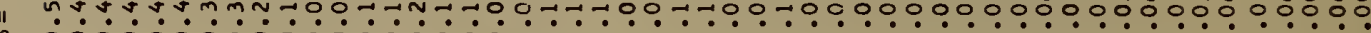

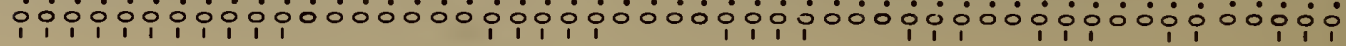

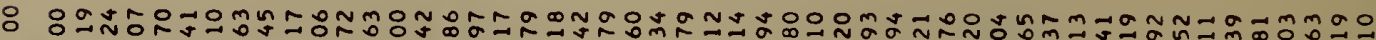

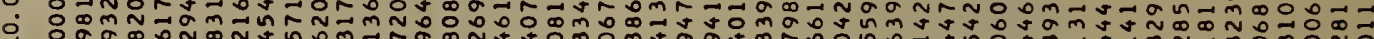

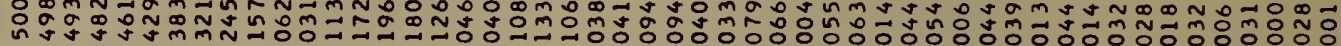

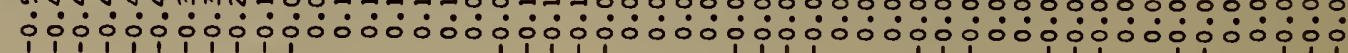
页

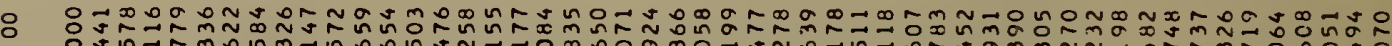

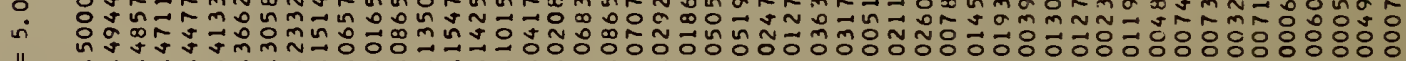

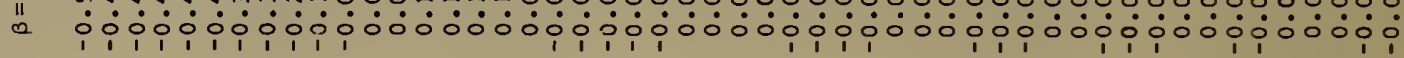

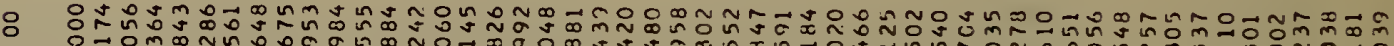
+

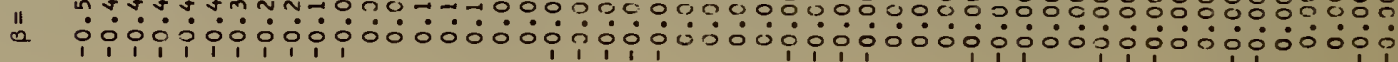

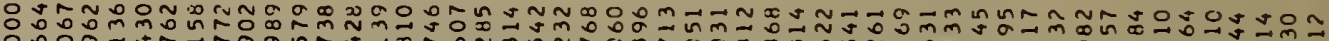

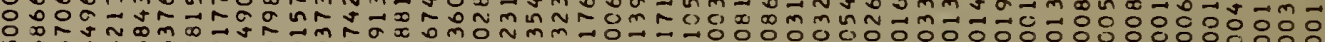

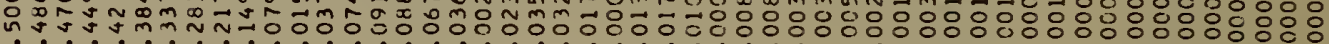

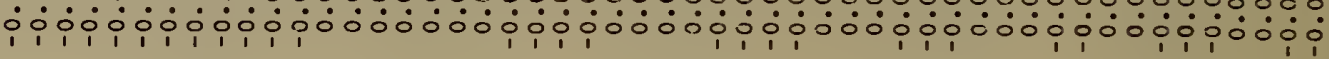

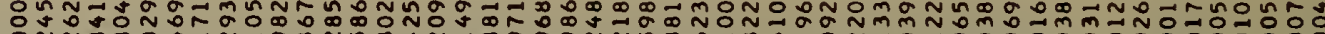

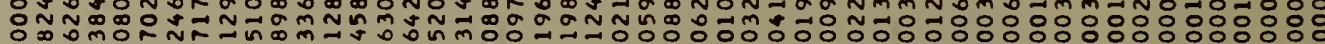

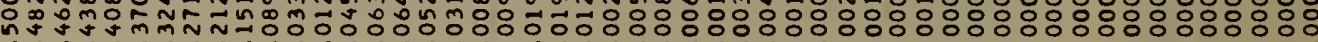

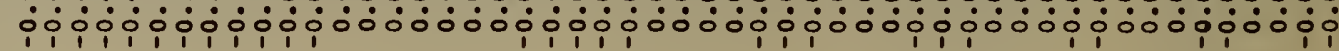

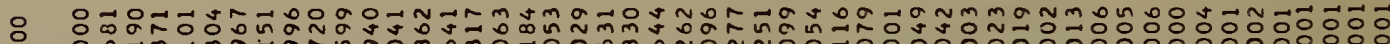
N :ํำ

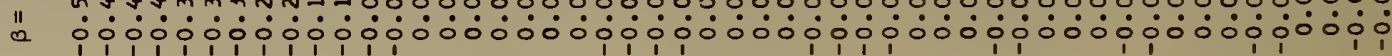

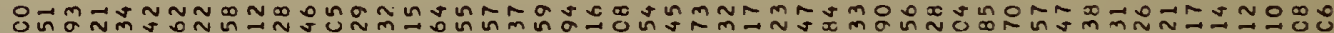

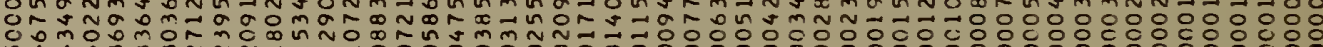 i}

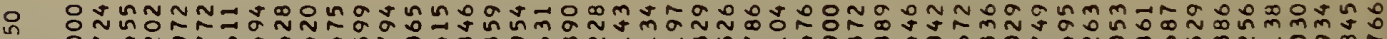

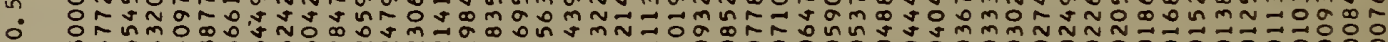

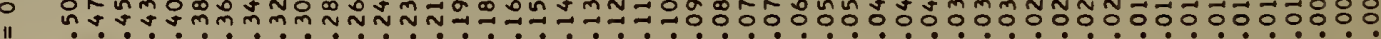

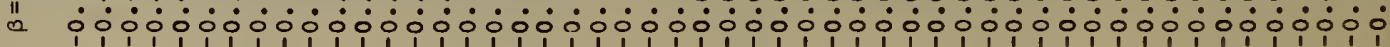
o 


\section{Appendix}

The purpose of this appendix is to describe the methods used to check the table entries. The Fresnel integral which was used throughout the calculations was evaluated by the method of Boersma [1960]. Boersma states that this method is suitable to evaluate the integral to eight decimal places over the whole range from 0 to $\infty$ with a small restriction which did not apply in these calculations.

Entries in tables $1 \mathrm{a}, 1 \mathrm{~b}, 2 \mathrm{a}$, and $2 \mathrm{~b}$ were evaluated using (6) with (4) for the linear aperture and (7) with (4) for the cubic aperture. The quantities used involved only Fresnel integrals, sines, and cosines in various combinations. The sines and cosines were evaluated by subroutines with a maximum error of $\pm 1\left(10^{-7}\right)$ and the Fresnel integral as described above. An investigation into the possible precision loss for both types of aperture tapers indicated that for the severest case this would be four digits at the very most. This loss occurred principally in the linear taper case for $\beta \leq 1$. This was verified by spot checking the tables for both types of apertures by differencing techniques.

The accuracy of the entires in tables $3 a$ and $3 b$ for the exponential taper was checked by increasing the number of Gaussian abscissas until the values for two arbitrarily chosen $n$ were essentially the same. For $n=96$ and 192 , the values for $\beta=5,10$ were exactly the same to five decimals. For $\beta=1,2,2.5,3$, and 4 , values of $\mathrm{n}=192$ and 384 gave the same results with a maximum difference of $\pm 1\left(10^{-5}\right)$. For $\beta=0.5$, the maximum difference for $\mathrm{n}=192$ and 384 was $\pm 1\left(10^{-5}\right)$ for $z_{0} \leq 4.1$. With the same $n$ values for $4.1<z_{0} \leq 5$, the maximum difference was $\pm 4\left(10^{-3}\right)$. However, for these few values, it did not seem feasible to repeat the calculations for larger $n$. The application of differencing techniques verified the above conclusions. 
For all three aperture types, if $\beta=\infty$,

$$
F \cong \int_{Z_{0}}^{\infty} e^{-\frac{i \pi}{2} z^{2}} d z=\frac{1-i}{2}-F_{0}\left(z_{0}\right) \text {, }
$$

where $F_{0}\left(Z_{0}\right)$ is the Fresnel integral $(9,10,11)$. The entries for $\beta=\infty$ in tables $3 a$ and $3 b$ were checked using the tables of Winjgaarden and Scheen [1949]. The values checked to the five decimals for all entires.

\section{Appendix References}

Boersma, J. (1960), On a numerical method for the computation of Fresnel integrals, Report TW2, Mathematics Institute, Univ. of Groningen.

Winjgaarden, Van A., and W. L. Scheen (1949], Table of Fresnel Integrals, Report R 49 of the computation department of the Mathematical Centre of Amsterdam. 



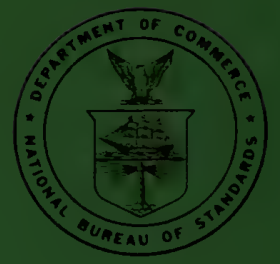

